\title{
Optical interference in nonlinear photonic crystals
}

\author{
Chien-Jen Lai, ${ }^{1,2}$ L.-H. Peng, ${ }^{2}$ and A. H. Kung ${ }^{1,3, *}$ \\ ${ }^{1}$ Institute of Atomic and Molecular Sciences, Academia Sinica, Taipei 10617, Taiwan \\ ${ }^{2}$ Department of Electrical Engineering and Institute of Photonics and Optoelectronics, National Taiwan University, \\ Taipei 10617, Taiwan \\ ${ }^{3}$ Department of Photonics, National Chiao-Tung University, Hsinchu 300, Taiwan \\ *Corresponding author: akung@pub.iams.sinica.edu.tw
}

Received July 18, 2007; revised September 22, 2007; accepted September 26, 2007; posted October 4, 2007 (Doc. ID 85438); published October 29, 2007

A model to analyze the interaction of the parametric fields being generated in a two-dimensional nonlinear photonic crystal has been developed. The analysis provides details of the interference of the generated wave(s) both inside and in the region just outside the crystal. The results are verified by second-harmonic generation in a $\mathrm{LiNbO}_{3}$ crystal that has been poled with a tetragonal inverted domain structure. (C) 2007 Optical Society of America

OCIS codes: $050.5298,190.2620,260.3160$.

Nonlinear photonic crystals have a homogeneous space-independent linear susceptibility $\chi^{(1)}$ but have a periodic or quasi-periodic two-dimensional (2D) spatial distribution of $\chi^{(2)}$ [1]. This 2D structure provides greater flexibility in the quasi-phase matching (QPM) [2] of wavelength conversion processes in a single crystal. As a consequence, simultaneous conversion of multiple wavelengths, multistep cascaded conversions, and conversion of broadband sources can be performed with good conversion [3-6]. Recently a general method for designing 2D nonlinear photonic crystals was proposed, allowing complete flexibility in designing photonic structures for simultaneous phase matching of arbitrary conversion processes [7].

Most of the analyses on nonlinear photonic crystals have focused on the QPM aspect, using an analogy to the 1D case and employing reciprocal lattices to picture the QPM process. Analysis of the propagation of coherent optical waves in these structures has been ignored. Intuitively, a 2D inverted domain structure can be viewed as having rows of $1 \mathrm{D}$ inverted domains aligned parallel to each other, each producing its own nonlinear wave signal [8]. This yields rows of coherent signal beams spaced exactly by the crystal's transverse domain period (Fig. 1). As these beams propagate, their diffraction results in optical interference among the beams. Since this happens inside the crystal, the interference occurs simultaneously with the nonlinear generation process. A full analysis of this has never been done. It is therefore interesting to develop a formalism to understand the nature of this interference. In this Letter we report for the first time to our knowledge a general mathematical description from the optical generation point of view optical interference in a nonlinear photonic crystal. We confirm the results of the analysis by a secondharmonic generation (SHG) experiment in a $2 \mathrm{D}$ crystal that has a tetragonal inverted domain structure.

We note that in nonlinear photonic crystals the input beam does not cause interference. The interference is of the signal waves that are being generated in the structure. As such, the effect is unique to nonlinear photonic crystals.
In our analysis we use real space representation to describe the propagation of the nonlinear signal waves through the medium. Diffraction of the waves is included to obtain a complete description of the field pattern both inside and outside the crystal. Both the fundamental and its second-harmonic $(\mathrm{SH})$ electric fields are linearly polarized along the $z$ axis (see Fig. 1), so they can be expressed in scalar form $E_{1}(\vec{r}, t)=U_{1}(\vec{r}) \exp (-i \omega t)$ and $E_{2}(\vec{r}, t)=U_{2}(\vec{r}) \exp (-i 2 \omega t)$, where the subscripts 1 and 2 stand for the fundamental and the SH, respectively, $\omega$ is the fundamental frequency, and $U_{1}(\vec{r})$ and $U_{2}(\vec{r})$ are complex amplitudes. The $\mathrm{SH}$ nonlinear polarization is given by $\widetilde{P}_{2}(\vec{r}, t)=P_{2}(\vec{r}) \exp (-i 2 \omega t)$, where $P_{2}(\vec{r})=\chi^{(2)}(\vec{r})\left[U_{1}(\vec{r})\right]^{2}$, and $\chi^{(2)}(\vec{r})$ is the local second-order nonlinear susceptibility.

An analytical expression for $U_{2}(\vec{r})$ can be obtained when $U_{1}(\vec{r})$ has a Gaussian profile:

$$
U_{1}(x, y, z) \approx \frac{U_{0}}{\left(1+x^{2} / b^{2}\right)^{1 / 2}} e^{-\left(y^{2}+z^{2}\right) / w^{2}} e^{-i \tan ^{-1}(x / b)} e^{i k_{1} x}
$$

where $\vec{r}$ has been replaced by the Cartesian coordinates $\vec{r}=(x, y, z) ; \quad w(x)=w_{0}\left(1+x^{2} / b^{2}\right)^{1 / 2}, \quad$ and $b$ $=k_{1} w_{0}^{2} / 2 . k_{1}$ is the propagation constant of the fundamental in the nonlinear material, $w_{0}$ is its beam
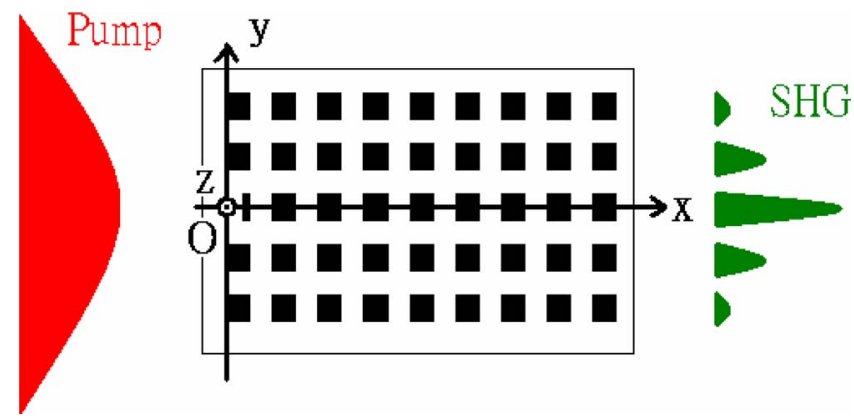

Fig. 1. (Color online) Periodical 2D QPM crystal depicted as the combination of several 1D QPM channels separated by unpoled channels of the same width. Black squares represent inverted domains. 
waist, and we assume $b$ is much greater than the length of the medium.

When $E_{1}$ and $E_{2}$ travel through a thin slab of thickness $\mathrm{d} x$, the small increment in $U_{2}(\vec{r})$ is [9]

$$
\mathrm{d} U_{2}(\vec{r})=\frac{i \mu_{0}(2 \omega)^{2}}{2 k_{2}} P_{2}(\vec{r}) \mathrm{d} x,
$$

where $k_{2}$ is the $\mathrm{SH}$ propagation constant in the nonlinear material and $\mu_{0}$ is the permeability of the material. When propagating from $\vec{r}^{\prime}$ to $\vec{r}$, this small increment evolves to become [10]

$$
\begin{aligned}
\mathrm{d} U_{2}\left(\vec{r}, \vec{r}^{\prime}\right)= & \frac{i \mu_{0}(2 \omega)^{2}}{2 k_{2}} \mathrm{~d} x^{\prime} \int F_{y} \exp \left[i G_{y}\right. \\
& \left.+i \sqrt{k_{2}^{2}-G_{y}^{2}}\left(x-x^{\prime}\right)\right] \mathrm{d} G_{y},
\end{aligned}
$$

where $F_{y}$ is defined as

$$
F_{y} \equiv \frac{1}{2 \pi} \int P_{2}(\vec{r}) e^{-i G_{y} y^{\prime}} \mathrm{d} y^{\prime}
$$

and $G_{y}$ is the reciprocal lattice constant in the $y$ direction. Here we neglect the diffraction effect in the $z$ direction because the domain polarization is uniform in $z$.

Integrating Eq. (3) over $x$ then gives

$$
\begin{aligned}
U_{2}(\vec{r})= & \frac{i 2 \mu_{0} \omega^{2}}{k_{2}} \int_{-\infty}^{x} \int F_{y}\left(G_{y}, x^{\prime}\right) \exp \left[i G_{y} y\right. \\
& \left.+i \sqrt{k_{2}^{2}-G_{y}^{2}}\left(x-x^{\prime}\right)\right] \mathrm{d} G_{y} \mathrm{~d} x^{\prime} .
\end{aligned}
$$

Equation (5) can be recognized as the integration of the wave vectors that are associated with the traveling Gaussian light waves and with the domain structure that is a 2D nonlinear grating in $\chi^{(2)}$ [1]. Numerically solving Eq. (5) gives the SH field amplitude at any point in the $x-y$ plane. Figure 2 shows the calculated $\mathrm{SH}$ intensity distribution in the $x-y$ plane generated in a $6 \mathrm{~mm}$ long crystal with a tetragonal domain structure for a fundamental beam entering at normal incidence. The crystal used has a poled domain period of $29.5 \mu \mathrm{m}$ in both the $x$ and $y$ directions designed for QPM SHG of $1990 \mathrm{~nm}$ at room tempera-

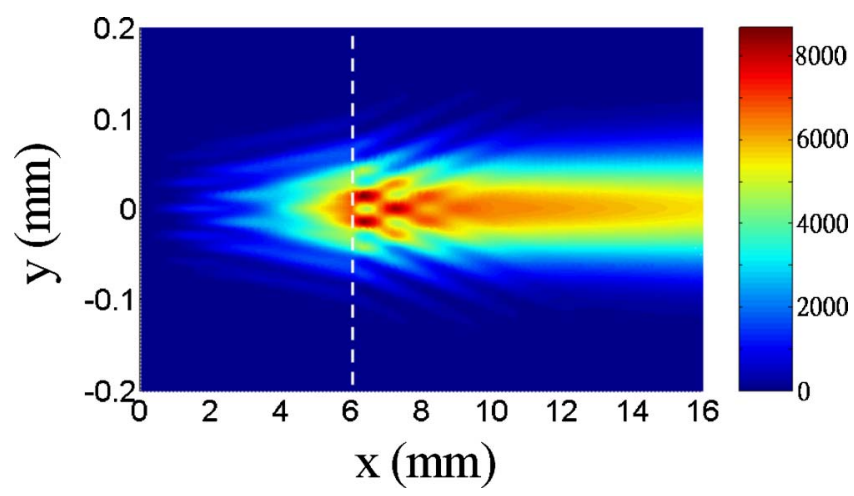

Fig. 2. (Color online) Simulated SH intensity distribution in the $x-y$ plane. $x$ is the distance from the input face of a nonlinear photonic crystal. The dashed line indicates the exit end of the crystal. ture in the forward $\left(\mathbf{G}_{10}\right)$ direction. The fundamental beam has a Gaussian waist of $100 \mu \mathrm{m}$ located at the center of the crystal. The corresponding Rayleigh length is $67 \mathrm{~mm}$. The Rayleigh length for light generated from a single channel of width $14.75 \mu \mathrm{m}$ is $\sim 770 \mu \mathrm{m}$. This is small compared with the crystal length. Hence the diffraction of the signal waves generated from each channel must be tracked during the simulation in the crystal. Figure 2 shows that the $\mathrm{SH}$ signal begins as separate beams generated from each 1D channel along the $x$ axis. These beams diffract as they grow and interfere with each other, creating an oscillatory transverse profile that evolves along the length of the crystal. The period of the interference pattern is equal to the transverse period of the nonlinear photonic structure. The resulting profile at the exit end of the crystal is not Gaussian and is clearly different from the Gaussian profile from a conventional SHG process. This interference due to the overlapping of coherent beams obviously affects the transverse beam profile at the output end of the crystal, and one can expect that this interference will also influence the phase-dependent generation process in an important way to result in a reduced conversion efficiency in the $2 \mathrm{D}$ structure as compared with the $1 \mathrm{D}$ case [11].

A SHG experiment was performed to validate this analysis. Pulsed radiation at $1990 \mathrm{~nm}$ from an optical parametric oscillator [12] was focused into the center of a PPLN crystal fabricated to have a 2D tetragonal distribution of poled domains with a period of $29.5 \mu \mathrm{m}$. The focused beam waist was $100 \mu \mathrm{m}$, and the incident IR intensity was $2 \mathrm{MW} / \mathrm{cm}^{2}$, which avoids pump depletion. The transverse spatial profile of the generated SH energy was image relayed with a $4 \times$ magnification and recorded by scanning in the $y$ direction across the magnified image a silicon photodetector equipped with a $10 \mu \mathrm{m}$ wide slit aperture. We used crystals of three different lengths, $l=1,2$, $4 \mathrm{~mm}$, to mimic three different distances inside the crystal in the simulation and recorded the images of the output at the exit end of each crystal. The measured profiles shown in Fig. 3 are in substantial agreement with the simulated results. As can be seen, interference begins to show at $x=\sim 1 \mathrm{~mm}$ (elevated baseline) and already becomes significant at $x=2 \mathrm{~mm}$.

Once outside the crystal, the optical interference is seen as that of a multibeam interference. Interference patterns persist near the crystal as a continuation of the interference from inside the crystal. The interference evolves into several component beams propagating into the far field, of $x \geq 12 \mathrm{~mm}$ in this example. The direction of each of these beams is given by a wave vector that satisfies the condition $k_{2, \mathrm{mn}}$ $=2 k_{1}+G_{\mathrm{mn}}$, consistent with the reciprocal lattice analysis employed in the literature [1]. In our example the $\mathbf{G}_{11}$ and $\mathbf{G}_{1,-1}$ directions are not phase matched. The SH intensity in these directions is much weaker, and the beams could hardly be seen. Yet as shown in Fig. 3 their contribution to the interference in the near field is still very significant. 


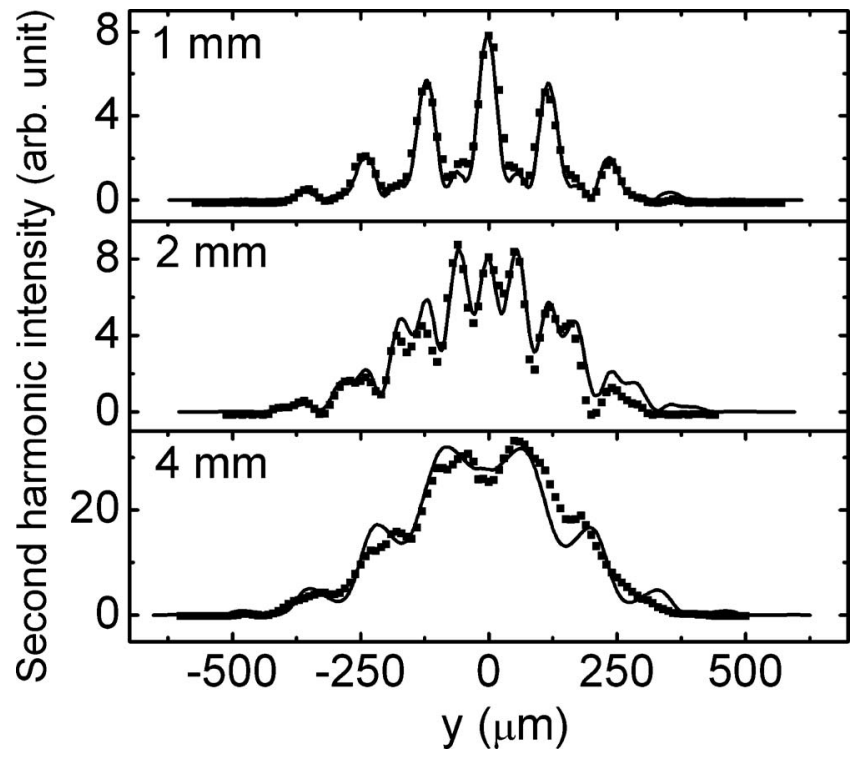

Fig. 3. Transverse SH intensity distribution at $x=1,2$, $4 \mathrm{~mm}$ from the input end of the crystal. The black dots are experimental points and the solid curves are simulated result. The horizontal scale is for $y$ in the image space, which is magnified four times by image relay relative to $y$ in Fig. 2.

Contrary to the $1 \mathrm{D}$ case, translation of a $2 \mathrm{D}$ nonlinear photonic crystal has a significant effect on the intensity pattern of the generated field because of the periodic poling pattern in the $y$ direction. Figure 4 shows the measured and the simulated $\mathrm{SH}$ signal at the exit end of two crystal lengths generated under the same experimental conditions described above. Here, the position of the apertured silicon photodetector was fixed while the crystal was translated in $1 \mu \mathrm{m}$ steps in the $y$ direction. Translating a $1 \mathrm{~mm}$ long crystal leads to a signal with the same periodic-

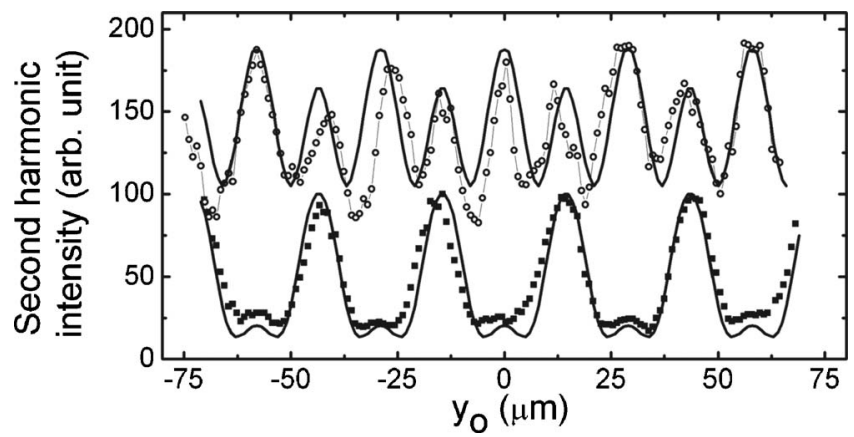

Fig. 4. Experimental (circles and squares) and simulated (solid curve) $\mathrm{SH}$ intensity at a fixed $y$ position when the crystal is translated a relative distance $y_{0}$ in the $y$ direction. The lower trace is for a $1 \mathrm{~mm}$ long crystal. The upper trace is for a $2 \mathrm{~mm}$ long crystal. They share the same vertical axis. $y_{0}=0$ is chosen as the location where the simulated signal is largest from the $2 \mathrm{~mm}$ crystal. ity as the channel period, and the signal reaches its peaks or valleys according to whether the detector is aligned to a poled or unpoled channel, respectively (see Fig. 1). Since the $1 \mathrm{~mm}$ crystal length is about the same as the Rayleigh length of $0.77 \mathrm{~mm}$ of each channel, the effect of interference results only in a small rise from the bottom of the valley in the signal, as can be seen in both the experiment and in the simulation. The interference effect is much more pronounced in the case of a $2 \mathrm{~mm}$ long crystal. In fact, in this case constructive interference results in an unanticipated larger signal corresponding to the detector aligned to an unpoled channel compared with when aligned to a poled channel. This type of interference phenomenon certainly is quite unique to nonlinear photonic crystals or similar types of structure.

The above examples serve to demonstrate that the analysis we have developed gives a general quantitative description of the interference effect of the $\mathrm{SH}$ field in a nonlinear photonic crystal. While the simulation is for SHG in a tetragonal domain structure, the approach can easily be generalized to many QPM processes such as cascaded harmonic generation and optical downconversion, and for arbitrary 2D and 3D domain patterns with an appropriate substitution of the description for $E_{2}$ and $\chi^{(2)}$ into the model.

We thank Chen-De Lin and Shih-Yu Tu for their valuable assistance. This work was supported by the National Science Council (NSC-95-2120-M-001-006) and by the Academia Sinica (AS-95-TP-A09).

\section{References}

1. V. Berger, Phys. Rev. Lett. 81, 4136 (1998).

2. J. A. Armstrong, N. Bloembergen, J. Ducuing, and P. S. Pershan, Phys. Rev. 127, 1918 (1962).

3. N. G. R. Broderick, G. W. Ross, H. L. Offenhaus, D. J. Richardson, and D. C. Hanna, Phys. Rev. Lett. 84, 4345 (2000).

4. A. H. Norton and C. M. de Sterke, Opt. Lett. 28, 188 (2003).

5. L.-H. Peng, C. C. Hsu, J. Ng, and A. H. Kung, Appl. Phys. Lett. 84, 3250 (2004).

6. N. G. R. Broderick, R. T. Bradfalean, T. M. Monro, D. J. Richardson, and C. M. de Sterke, J. Opt. Soc. Am. B 19, 2263 (2002).

7. R. Lifshitz, A. Arie, and A. Bahabad, Phys. Rev. Lett. 95, 133901 (2005)

8. J. R. Kurz, A. M. Schober, D. S. Hum, A. J. Salzman, and M. M. Fejer, IEEE J. Sel. Top. Quantum Electron. 8, 660 (2002).

9. G. D. Boyd and D. A. Kleinman, J. Appl. Phys. 39, 3597 (1968).

10. J. W. Goodman, in Introduction to Fourier Optics (McGraw Hill, 1996), Chap. 3.

11. L.-H. Peng, Chao-Ching Hsu, and A. H. Kung, IEEE J. Sel. Top. Quantum Electron. 10, 1142 (2004).

12. C. S. Yu and A. H. Kung, J. Opt. Soc. Am. B 16, 2233 (1999). 